\title{
Nerve Sheath Myxoma (Neurothekeoma) of the Gingiva, A Case Report and Review of the Literature
}

\author{
Rima A. Safadi • John W. Hellstein • \\ May M. Diab $\cdot$ Huda M. Hammad
}

Received: 22 March 2010/Accepted: 30 April 2010/Published online: 26 May 2010

(c) Humana 2010

\begin{abstract}
Nerve sheath myxoma (NSM) is a benign peripheral nerve sheath tumor that rarely occurs in the oral cavity. Among the 23 reported intraoral cases, no lesion has previously been reported on the gingiva. In this report, we describe the first gingival case of oral neurothekeoma with histopathologic and immunohistochemical characteristics. The patient, a 32 year old female presented with a slowly growing gingival mass diagnosed clinically as an epulis. The lesion was surgically excised. Histopathologically, the lesion presented as a submucosal multinodular mass composed of spindle and stellate-shaped cells with a myxoid background. Immunohistochemically, the tumor cells were sporadically positive for S-100 and NSE and negative for GFAP, EMA, SMA, CD68 and HMB45. The immunoprofile of this lesion confirmed a Schwann cell origin. The lesion was followed up for 10 months with no reports of recurrence.
\end{abstract}

Keywords Nerve sheath myxoma $\cdot$ Neurothekeoma . Gingiva $\cdot$ Oral cavity $\cdot$ Benign tumor

R. A. Safadi $(\bowtie) \cdot$ H. M. Hammad

Department of Oral Surgery and Medicine, Faculty of Dentistry, Jordan University of Science and Technology, P. O. Box 3030, Irbid 22110, Jordan

e-mail: rsafadi@just.edu.jo; rimaasafadi@yahoo.com

R. A. Safadi - M. M. Diab - H. M. Hammad

King Abdullah University Hospital, Irbid, Jordan

J. W. Hellstein

Oral Pathology, Radiology and Medicine Department, College

of Dentistry, The University of Iowa, Iowa City, IA, USA

\section{Introduction}

Nerve sheath myxoma (neurothekeoma) is an uncommon benign soft tissue tumor of peripheral nerves. The lesion was first described in 1969 by Harkin and Reed and was called nerve sheath myxoma [1]. In 1980 Gallagher and Helwig used the term neurothekeoma to describe the same tumor [2]. Although the lesion is often located in the dermis and subcutaneous tissues of the head and neck region it is rarely seen intraorally [3]. A computer assisted PubMed® search revealed only 23 reported intraoral cases of NSM. The age of reported oral cases ranged from newborn to 73 years old $[4,5]$. There may be a slight female predilection with a ratio of 13:9 on the 22 cases where sex was reported. The maximum diameter of lesions ranged from $0.5 \mathrm{~cm} \mathrm{[6]} \mathrm{to} 3.0 \mathrm{~cm}$ [4]. The most common intraoral site is the tongue (10/23) followed in decreasing order by buccal mucosa (7/23), palate (3/23), lower lip (2/23) and retromolar pad (1/23). The appearance of nerve sheath myxoma presenting as a gingival epulis has not been reported. This study reports the clinical, histopathologic and immunohistochemical features of the first reported case of a gingival nerve sheath myxoma.

\section{Case Report}

A 32-year-old female presented to the dental clinic of Prince Basma Hospital complaining of a painless, slowly enlarging mass over 6 months of the labial marginal gingiva of the upper right central incisor. The patient was not pregnant and with unremarkable family and medical histories. The oral surgeon excised the lesion under local anesthesia with a provisional clinical diagnosis of "epulis". 
Gross examination of the submitted specimen revealed a reniform, moderately firm elastic nodule measuring $0.8 \times 0.6 \times 0.6 \mathrm{~cm}$. The surface was tan and smooth. Cut surfaces revealed subepithelial homogenous grey tissue with a soft consistency.

Histopathologic examination of hematoxylin and eosin stained sections revealed a submucosal, well-demarcated mass with lobular arrangement. The lobules were of loosely arranged cells though focal areas displayed closely clustered thin spindled and stellate cells mixed with variable amounts of myxoid matrix (Fig. 1). Occasionally, a swirling growth pattern was seen in the more cellular areas. Short thick collagen bundles as well as mast cells were interspersed within the lobules (Fig. 2). Immunohistochemical stains were conducted for S100 protein, neuron specific enolase (NSE), epithelial membrane antigen (EMA), glial fibrillary acidic protein (GFAP), CD68 (a macrophage marker), smooth muscle actin (SMA) and

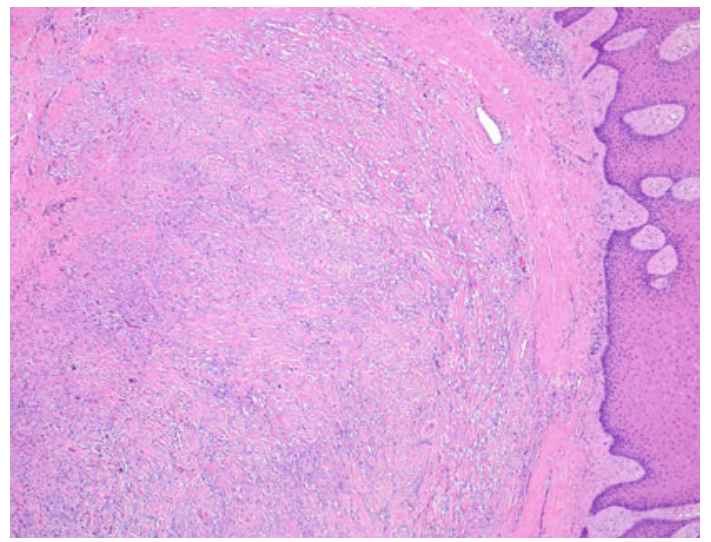

Fig. 1 Hematoxylin and eosin stain of neurothekeoma section $(10 \times)$. Notice the well demarcation of the lesion from superficial submucosa

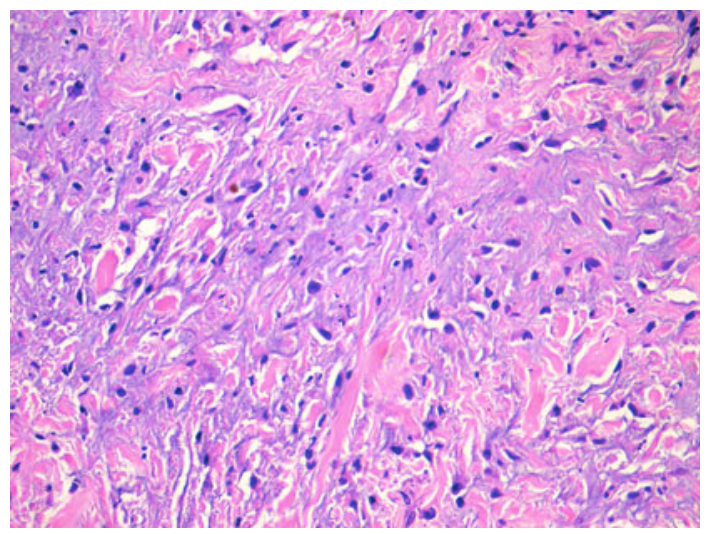

Fig. 2 Hematoxylin and eosin stain of neurothekeoma sections $(20 \times)$. Note the presence of thick, short collagen bundles and focal myxoid areas. Note the presence of epithelioid/stellate cells

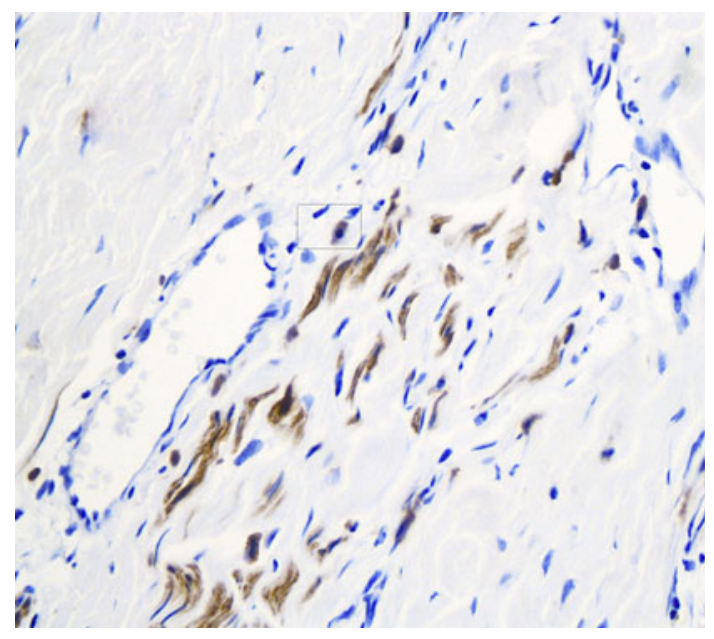

Fig. 3 Immunohistochemical stain of S100 protein counterstained with hematoxylin, using horse radish peroxidase technique $(40 \times)$. Note the positivity of thin spindled neural cells in short fascicles presented as brown/darkly stained cytoplasm. Note the presence of a mast cell in the rounded rectangle

HMB45 (a melanocyte marker). Figure 3 shows clusters of S100 positive, wavy, thin spindled cells with scattered mast cells, sometimes with close proximity to neural cells. Figure 4 presents an image of NSE section that shows spindled and stellate lesional cells with variable positivity. Tumor cells were negative for GFAP, CD68, SMA and HMB45.

Based on the histopathologic and immunohistochemical results, the case was diagnosed as nerve sheath myxoma of the mixed type.

The patient was followed up for 10 months with no reports of recurrence.

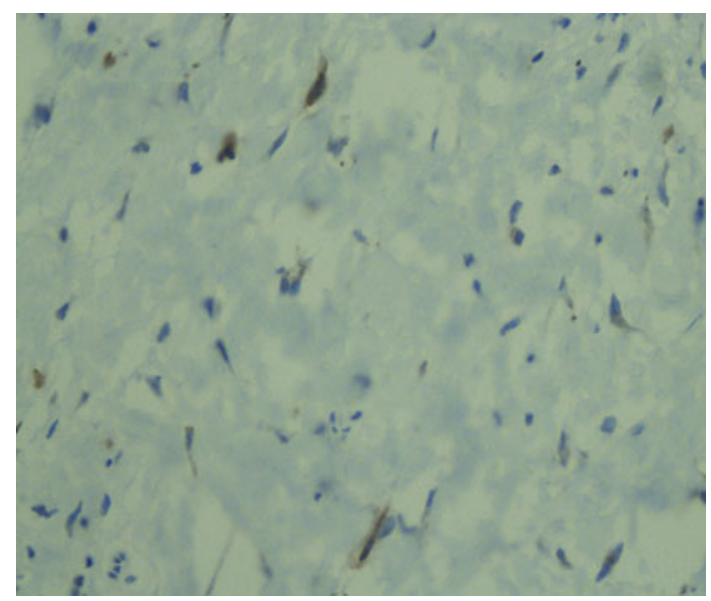

Fig. 4 Immunohistochemical stain of NSE protein counterstained with hematoxylin, using horse radish peroxidase technique $(40 \times)$. Note the positivity of some of spindled and stellate tumor cells presented as brown/darkly stained cytoplasm 


\section{Discussion}

Oral nerve sheath myxoma is a relatively rare benign oral tumor. Nishioka et al., reported only three oral cases of neurothekeoma out of 12,635 diagnosed cases contained in their archives [3]. Reviewing the literature, NSM has not been reported in oral locations other than tongue, buccal mucosa, palate, lip and retromolar pad. This study reports the first case of NSM (neurothekeoma) that occurred on the marginal gingiva simulating an epulis.

Based on the amount of myxoid stroma, nerve sheath myxoma is generally divided into myxoid (classical), mixed and cellular types [7]. Neurothekeoma is the term used to represent the cellular variant [2, 8] while nerve sheath myxoma is used to describe the less cellular but more myxoid variant [1]. The case reported in this study is of the mixed type showing moderate amounts of myxoid stroma.

Reviewing the reported cases of oral nerve sheath myxoma (neurothekeoma), a consistent immunohistochemical profile of the three histopathologic variants has not been established. Schortinghuis [5] reported a myxoid case of nerve sheath myxoma which was negative for S100 while the myxoid NSM cases reported by Nishioka [3] and Makino [9] were S100 and NSE positive. Penarrocha [4] and Nishioka et al. [3] reported two mixed-type cases, which were positive for both S100 and NSE. The diagnosis of the cellular variant was given for one of the three oral cases reported by Nishioka et al. [3]. That cellular lesion was both S100 and NSE positive. The other two cases reported by Nishioka et al. [3] were of classic and mixed variants and all showed the same S100 and NSE immunoprofile.

The cell of origin of NSM is controversial with theories proposing a proliferation of Schwann cells or other perineural cells [10]. Schwann cells are generally positive for S100 and NSE while negative for EMA, [11, 12]. In contrast cells of perineural origin display S100 negativitiy and EMA [12]. Fetsch et al. postulated a tendency of NSM to recruit histiocytic cells and to differentiate into myofibroblasts [13]. SMA positivity suggests some similarity to the epithelioid variant of leiomyoma or myofibroblastic proliferations [14]. GFAP positivity is rare in NSM; however, its positivity further supports a peripheral nerve sheath origin as occasional peripheral nerve sheath tumors may co-express GFAP and S100 [15].

The immunohistochemical profile of the case presented in this study is of S100 positivity, weak positivity for NSE, negativity for GFAP, EMA, CD68, SMA and HMB45 confirming nerve sheath origin from Schwann cells and excluding perineural, histiocytic, myofibroblastic, leiomyomatous and melanocytic origins.

The clinical differential diagnoses of NSM presenting as an epulis include chronic hyperplastic gingivitis, focal fibrous hyperplasia, pyogenic granuloma, peripheral ossifying fibroma, peripheral giant cell granuloma, giant cell fibroma and peripheral odontogenic fibroma.

The histopathologic differential diagnosis of nerve sheath myxoma includes neural and non-neural lesions. Neural lesions include neurilemmoma, true neuroma and myxoid neurofibroma. Neurilemmomas are encapsulated with Antoni A tissue that shows Verocay bodies. Though the Antoni B areas of neurilemmomas are myxoid the overall features of neurilemmomas are not seen in NSM. True neuromas exhibit the presence of nerve axons. Neurofibromas, when myxoid, exhibit loose myxoid component in the fascicles and in the surrounding tissue and are generally not well-demarcated. The cells are more slender and closely aggregated. Non neural lesions within the histologic differential include oral focal mucinosis and soft tissue myxoma. Mast cells are seen in NSM but not in oral focal mucinosis. Likewise, oral focal mucinosis shows well-circumscribed lesion with uniform appearance and do not show the immunoprofile of the NSM. Additionally, the pattern of NSM is lobular with condensed fibrous tissue at the periphery. Mast cells are not a feature of soft tissue myxomas which show blending with the surrounding tissues in contrast to NSM which is well demarcated from surrounding tissue.

Local excision is the treatment of choice for NSM. No recurrence after local excision has been reported for oral cases.

In summary, this case report describes the 24th reported case of oral nerve sheath myxoma and the first gingival case presenting as an epulis.

\section{References}

1. Harkin JC, Reed JJ. Tumors of the peripheral nervous system, second series, Fasc, 3. Washington, D. C: Armed Forces Institute of Pathology; 1969. p. 60-4.

2. Gallagher RL, Helwig EB. Neurothekeoma-a benign cutaneous tumor of neural origin. Am J Clin Pathol. 1980;74:759-61.

3. Nishioka M, Aguirre RL, Ishikawa A, et al. Nerve sheath myxoma (neurothekeoma) arising in the oral cavity: histological and immunohistochemical features of 3 cases. Oral Surg Oral Med Oral Pathol Oral Radiol Endod. 2009;107:e28-33.

4. Peñarrocha M, Bonet J, Minguez JM, et al. Nerve sheath myxoma (neurothekeoma) in the tongue of a newborn. Oral Surg Oral Med Oral Pathol Oral Radiol Endod. 2000;90:74-7.

5. Schortinghuis J, Hille JJ, Singh S. Intraoral myxoid nerve sheath tumour. Oral Dis. 2001;7:196-9.

6. Sist TC, Green GW. Benign nerve sheath myxoma: light and electron microscopic features of two cases. Oral Surg Oral Med Oral Pathol. 1979;47:441-4.

7. Argenyi ZB, LeBoit PE, Cruz DS, et al. Nerve sheath myxoma (neurothekeoma) of the skin: light microscopic and immunohistochemical reappraisal of the cellular variant. J Cutan Pathol. 1993;20:294-303. 
8. Tiffee JC, Pulitzer DR. Nerve sheath myxoma of the oral cavity: case report and review. Oral Surg Oral Med Oral Pathol Oral Radiol Endod. 1996;82:423-5.

9. Makino T, Utsunomiya T, Kamino Y, et al. Nerve sheath myxoma of the tongue in a child. Int $\mathrm{J}$ Oral Maxillofac Surg. 2002;31:451-4.

10. Yamashaita N, Minami S, Yu M. Dermal nerve-sheath myxoma. J Dermatol. 1990;17:564-8.

11. Angervall L, Kindblom LG, Haglid K. Dermal nerve sheath myxoma: a light and electron microscopic, histochemical and immunohistochemical study. Cancer. 1984;53:1752-9.

12. Perentes E, Nakagawa Y, Ross GW, Stanton C, Rubinstein LJ. Expression of epithelial membrane antigen in perineural cells and their derivatives. Acta Neuropathol (Berl). 1987;75:160-5.
13. Fetsch JF, Laskin WB, Hallman JR, et al. Neurothekeoma: an analysis of 178 tumors with detailed immunohistochemical data and long-term patient follow-up information. Am J Surg Pathol. 2007;31:1103-4.

14. Calonje E, Wilson-Jones E, Smith NP, et al. Cellular 'neurothekeoma': an epithelioid variant of pilar leiomyoma. Morphological and immunohistochemical analysis of a series. Histopathology. 1992;20:397-404.

15. Katsourakis M, Kapranos N, Papanicolaou SI, et al. Nerve-sheath myxoma (neurothekeoma) of the oral cavity: a case report and review of the literature. J Oral Maxillofac Surg. 1996;54:904-6. 